\title{
A Nonlinear Instrument Diaphragm
}

\author{
Fidel Cordero, Harry Matheson, and Daniel P. Johnson
}

\begin{abstract}
Details of fabrication for the production of sensitive diaphragms having a controlled nonlinear pressure-deflection characteristic are presented. The desired characteristic was such that when the diaphragm formed one plate of a condenser in the frequency-controlling network of a Wien-bridge oscillator, the resulting pressure-frequency transfer characteristic would be linear between -30 and +30 dynes per square centimeter. Typical transfer curves are shown.
\end{abstract}

\section{Introduction}

The diaphragm described herein was required to have a deflection versus pressure characteristic such that, with the diaphragm as the moving plate of a condenser inserted in the frequency-controlling circuit of a Wien-bridge oscillator, the frequency deviations of the oscillator would be proportional to the pressure difference across the diaphragm. For a Wien-bridge oscillator the frequency-capacitance relation for variation of one capacitor is $f \propto C^{-1 / 2}$. Assuming an ideal parallel-plate condenser, the wanted pressure-displacement relation over the working range of the diaphragm was $p \propto d^{+1 / 2}$. The imperfect inverse proportionality between capacitance and plate separation in a physically realizable condenser necessitated some empirical adjustment of the diaphragm configuration to obtain the required characteristic.

Additional specifications for the diaphragm-oscillator combination called for a sensitivity of $0.3-$ to 1.0-percent frequency change per dyne per square centimeter, good linearity between -30 and +30 $\mathrm{d} / \mathrm{cm}^{2}$, and good long-time stability. In addition, the diaphragm was required also to tolerate momentary overpressure of the order of $1,000 \mathrm{~d} / \mathrm{cm}^{2}$.

\section{Description of Diaphragm}

\subsection{Forming Die}

The diaphragms were pressed from 0.001-in.-thick commercial soft-brass shim stock. Figure 1 shows the arrangement of the 4 circumferential and 60 radial corrugations impressed in a finished diaphragm. Not discernible in the photograph is the slight concavity, or cupping, imparted to the diaphragm during the forming operation.

The combined effect of the radial corrugations and concavity compels the diaphragm to execute an umbrella-like motion when pressure is applied. It is more compliant when pressure is applied to its convex side than to its concave side.

Figure 2 gives the essential dimensions of the die. Each radial groove was 0.015 in. deep at its outer end and 0.005 in. deep at its inner end, and the central areas were depressed 0.005 in. with respect to the circumferential flats. Although the grooves in the die were cut with a sharp $V$-pointed tool, the forming pressure was not great enough to force the metal to the bottom of the grooves. As a result, the cross

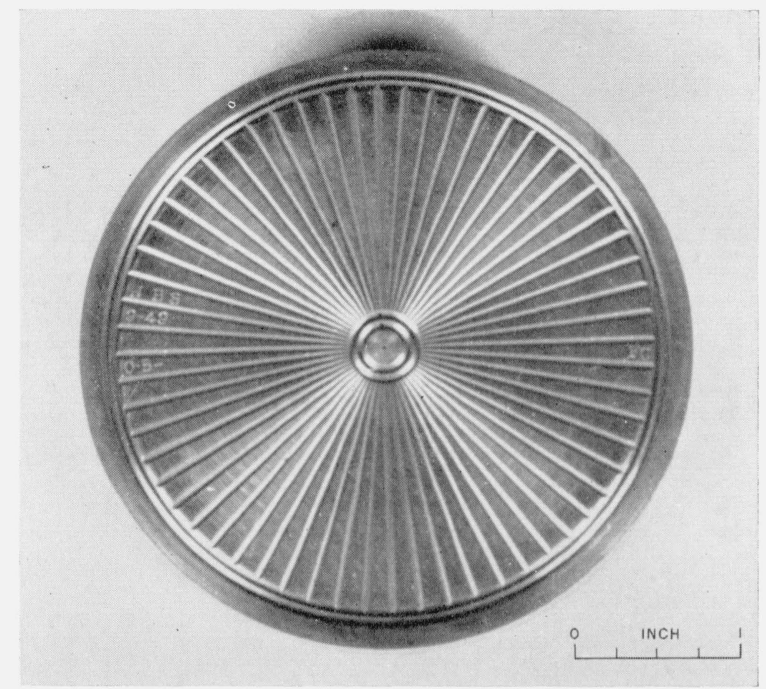

Figure 1. Matrix for forming a nontinear diaphragm.

sections acquired by the corrugations were nearly arcs of circles.

Earlier dies had a single, small hole at the center to allow escape of air during the forming operation. Later dies were provided with holes for each circumferential groove. In addition, the edges of the circumferential grooves of later dies were rounded to reduce the tendency of the sheet metal to tear during the forming operation. This rounding also resulted in more uniform characteristics in the finished diaphragms. The surface of the die, including the grooves, was brought to a medium polish.

\subsection{Diaphragm Fabrication}

Annealed brass shim stock, commercially available in rolls 6 in. wide, was first examined for pinholes under back lighting. Disks, somewhat greater than $3 \frac{1}{2}$ in. in diameter, were cut out of pinhole-free areas with scissors, using care not to kink the material. Similar disks were cut from unbleached paper. Two of the brass and three paper disks were then interleaved, clamped between brass blocks in a lathe, and turned to the final diameter.

The diaphragms were formed by pressing them against the clean oilfree die in a hydraulic press arranged as shown in figures 3 and 4 . One of the 


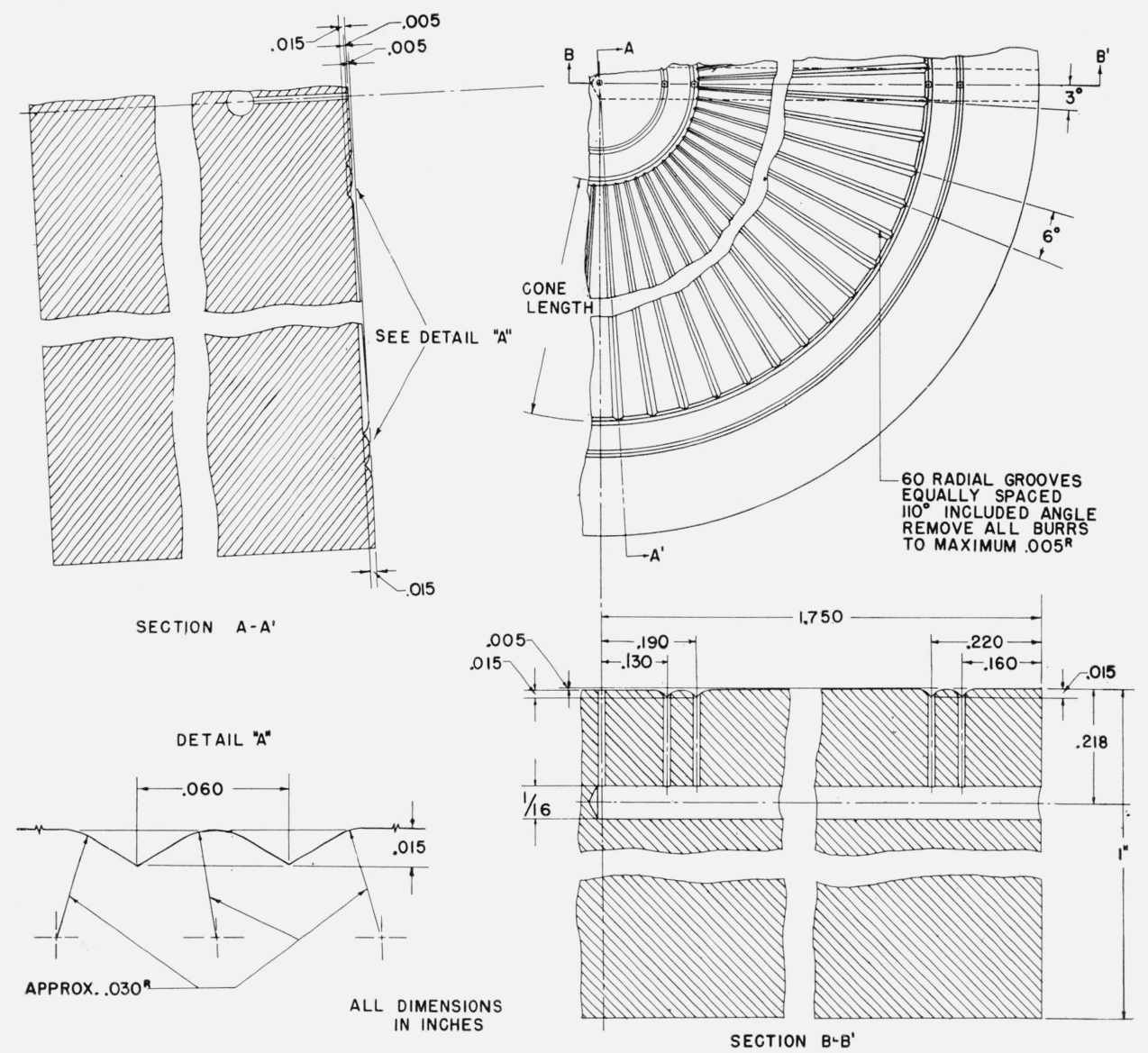

Figure 2. Drawing for reproducing a matrix.

Note that each radial groove is $0.015 \mathrm{in}$. deep at its outer end and $0.005 \mathrm{in}$. deep at its inner end and that the central areas are depressed $0.005 \mathrm{in}$. with respect to the circumferential flats.

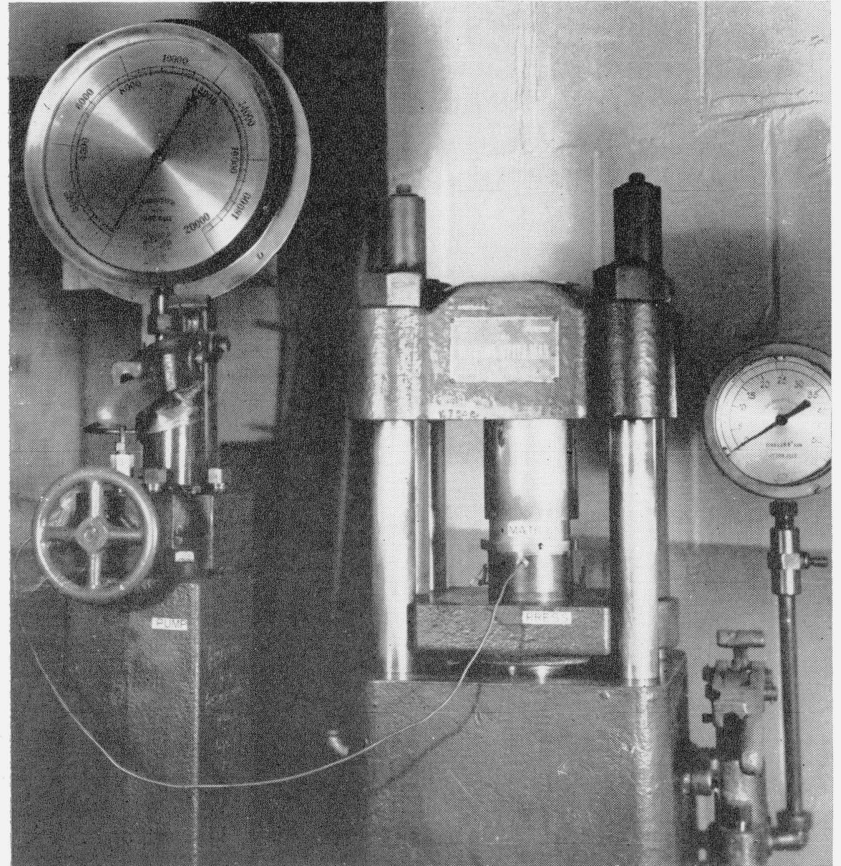

FIGURE 3. Hydraulic press arranged for forming diaphragms,
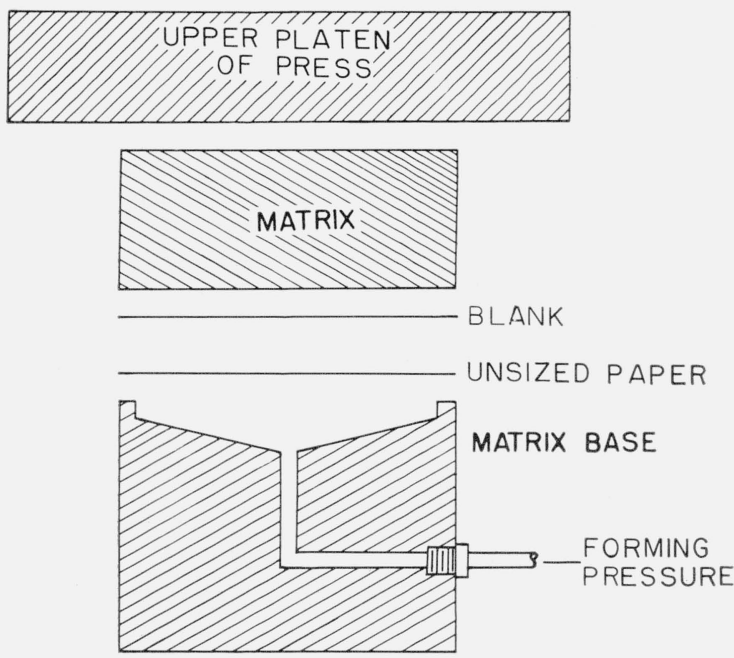

LOWER PLATEN

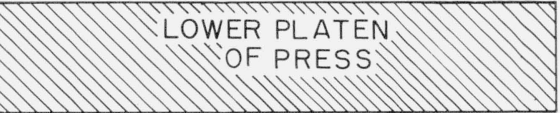

FIGURE 4. Original arrangement of equipment for forming diaphragms. 
paper disks was used as a gasket to seal the joint between the lower platen and the diaphragm. After the press was closed with a force of 10 tons concentrated on an annulus $0.170 \mathrm{in}$. wide at the edge of the diaphragm, the diaphragm was formed by the application of 1,500 psi for several seconds.

After release of the forming pressure, the press was opened and the die was removed. Absence of oil on the face of the die indicated that the diaphragm was not torn during the forming process. The diaphragm and paper gasket were carefully slid off the annulus of the lower platen and the gasket peeled from the diaphragm. The diaphragm was rinsed in petroleum ether, using a wire holder, carefully examined for pinholes and tears under back lighting, and stored in a cardboard rack.

More recently the fabrication procedure has been modified either as a matter of convenience or because it was felt that a more uniform product resulted. The modifications were

(1) The blanks were cut without paper interleaves, turning the lathe by hand and using a razor blade as a cutting edge.

(2) Rolled edges and burrs on the disks were removed by laying the blank on a piece of plate glass and dragging a razor blade over the edge of the blank to straighten the edge.

(3) The blank was protected from the hydraulic fluid by a sheet of dental dam. The dam was clamped between brass rings and was held over the bottom platen. The rings also served to center the blank and die for forming.

(4) Lens paper was substituted for the bulkier, unsized paper and placed between the blank and the dental dam.

(5) After forming the diaphragm it was removed from the recess formed by the brass rings and dental dam by gently touching its edge with scotch tape and lifting it from the recess.

(6) The petroleum ether dip was omitted.

Once a diaphragm was formed, it became essential not to handle it by the edge or in any manner that would produce distortion across a chord or diameter.

\section{Proof Tests}

The diaphragms were formed against a die with the center recessed 0.005 in. When the forming pressure was removed, the central part of the diaphragm sprang away from the die, so that the center of the diaphragm was above the plane of the rim, i. e., the diaphragm acquired a cupped shape.

\subsection{Cupping and Compliance}

For measurement of cupping and compliance, the diaphragms were mounted in a jig consisting of two brass disks with clamping rings cut on their mating surfaces. The jig was mounted to hold the diaphragm horizontally on the carriage of a milling machine. The position of the diaphragm was detected by a fixed-wire probe connected to an electronic indicator (capacitance bridge). The diaphragm was brought into contact with the probe by moving the carriage vertically or horizontally by the positioning screws of the carriage. Displacements were measured with the micrometers on the positioning screws.

Most of the diaphragms showed a cupping of 0.004 to 0.008 in., with occasional values as low as 0 and as high as $0.015 \mathrm{in}$. or more. As no direct relation between cupping and either sensitivity or dynamic range was observed, diaphragms whose cupping was within the range 0.004 to 0.008 in. were considered acceptable for further testing.

The mechanical compliance of each diaphragm was measured by determining the pressure necessary to produce central displacements of $+0.010,+0.005$, -0.005 and $-0.010 \mathrm{in}$. of the diaphragm. Some typical values are shown in table 1 . A water-filled manometer with a 1:20 slope was adequate for determining the pressure.

TABLE 1. Mechanical compliance of representative diaphragms

\begin{tabular}{|c|c|c|c|c|c|}
\hline \multirow{2}{*}{ Diaphragm } & \multicolumn{5}{|c|}{ Pressure for the following deflections in inches ${ }^{1}$} \\
\hline & +0.010 & +0.005 & 0 & -0.005 & -0.010 \\
\hline $\begin{array}{l}208 . . \\
170 . \\
226 . \\
222 \\
162 .\end{array}$ & $\begin{array}{r}d / \mathrm{cm}^{2} \\
+134 \\
+125 \\
+124 \\
+135 \\
+118\end{array}$ & $\begin{array}{l}d / \mathrm{cm}^{2} \\
+53 \\
+47 \\
+49 \\
+52 \\
+45\end{array}$ & $\begin{array}{c}d / c m^{2} \\
0 \\
0 \\
0 \\
0 \\
0\end{array}$ & $\begin{array}{c}d / c m^{2} \\
-38 \\
-35 \\
-37 \\
-38 \\
-31\end{array}$ & $\begin{array}{l}d / c m^{2} \\
-67 \\
-61 \\
-64 \\
-66 \\
-53\end{array}$ \\
\hline $\begin{array}{l}249 \\
33 \\
14 \\
10 \\
9\end{array}$ & $\begin{array}{l}+134 \\
+144 \\
+130 \\
+142 \\
+144\end{array}$ & $\begin{array}{l}+51 \\
+52 \\
+47 \\
+54 \\
+53\end{array}$ & $\begin{array}{l}0 \\
0 \\
0 \\
0 \\
0\end{array}$ & $\begin{array}{l}-37 \\
-33 \\
-32 \\
-34 \\
-36\end{array}$ & $\begin{array}{l}-64 \\
-52 \\
-49 \\
-57 \\
-58\end{array}$ \\
\hline
\end{tabular}

1 The diaphragm moves toward the backplate for a positive pressure difference.

The mechanical compliance was not found to be directly related to the observed sensitivity or dynamic range of a finished capsule, probably because of the effect of clamping pressure discussed below. However, the measurements did serve to reject diaphragms that were obviously too stiff, too compliant, or for which the curvature of the load-deflection curve was obviously wrong. It was occasionally surprising to see two diaphragms with quite similar mechanical compliances result in finished capsules, one of which would be quite satisfactory, whereas the other showed only a limited linear range.

\subsection{Pressure-Frequency Relation}

After completion of the mechanical tests the diaphragm was mounted in its permanent holder, or capsule, as shown in figure 5, and the backplate was

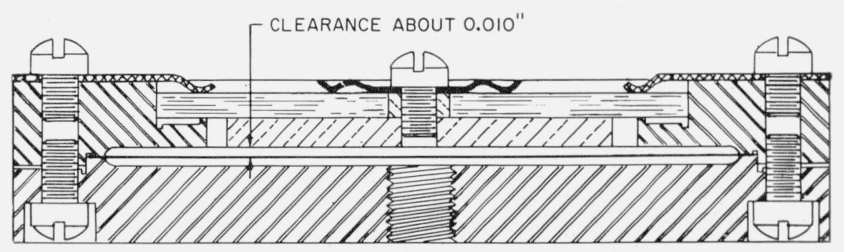

SECTION THROUGH CAPSULE

Figure 5. Cross-sectional sketch of assembled capsule showing the relative positions of the diaphragm and backplate. 


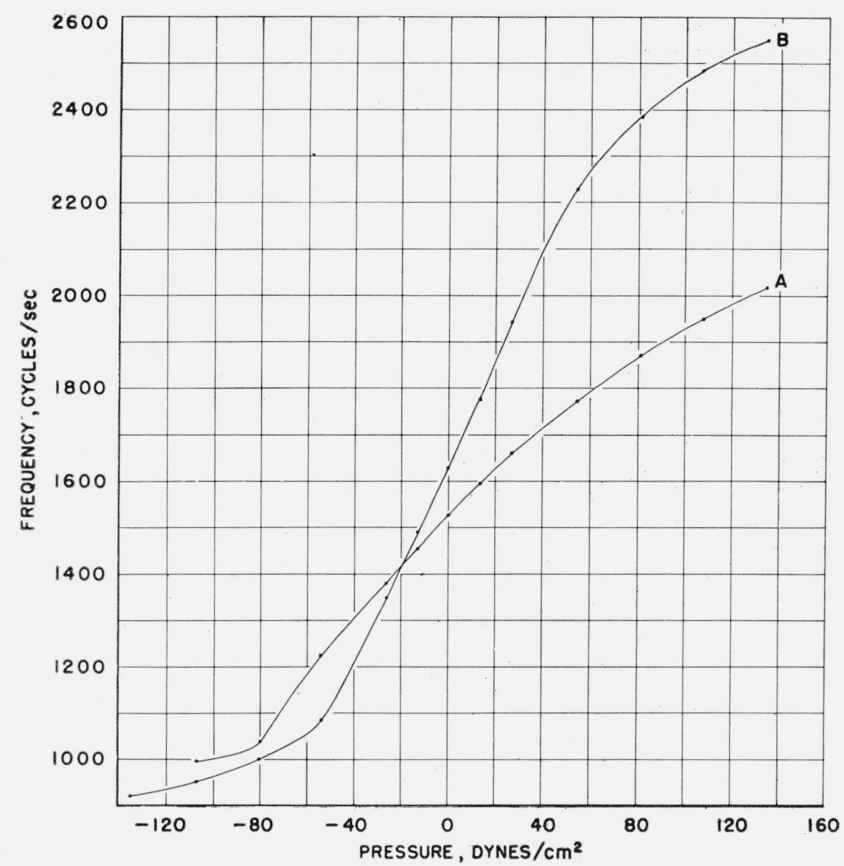

Figure 6. Pressure-frequency relations for capsule-oscillator combinations with adequate linear range.

A, Minimum acceptable sensitivity; B, excellent sensitivity. The diaphragm moves away from the backplate for a positive pressure difference.

shimmed to give an electrical capacitance of $100 \pm 5$ $\mu \mu \mathrm{f}$. Final acceptance of a diaphragm was based on a plot of the pressure difference across the diaphragm in its own capsule against the frequency of a Wienbridge oscillator connected to the capsule. For these measurements the pressure differences were obtained with a gas-pressure balance.

Calibration curves for two diaphragms with extreme characteristics are shown in figure 6. Except when intended for special purposes, the minimum acceptable linear range was from -25 to $+25 \mathrm{~d} / \mathrm{cm}^{2}$ and the acceptable range of sensitivities in the linear region was 5 to $11 \mathrm{cps}$ per $\mathrm{d} / \mathrm{cm}^{2}$.

\section{Capsule}

The diaphragm was clamped in a capsule body consisting of two heavy-brass rings, a backplate, an insulating glass plate, and miscellaneous screws and fittings. Figure 7 shows the parts in an exploded view. The three critical surfaces were the two that clamp the edge of the diaphragm and that which positions the glass plate in the top half of the capsule body, figure 5. Where it was necessary to seal the interface between the glass plate and capsule body and the threads of the backplate against leaks of air, a silicone grease was found to be satisfactory.

To avoid electric short circuits in a capsule when the diaphragm was subjected to excessive pressure, the exposed surface of the backplate was coated with an insulating lacquer.

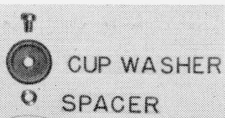

GLASS PLATE

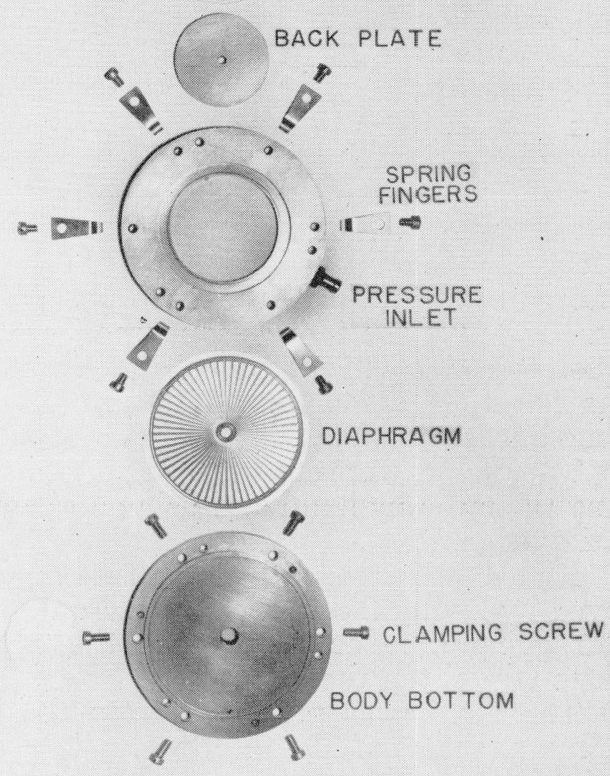

Figure 7. Parts of a complete capsule for comparison with figure 5.

The two halves of the capsule body were held together by six 10-32 brass screws. A study of the optimum clamping pressure showed, surprisingly enough, that very small distortions of the capsule body were often essential to obtain optimum characteristics.

\subsection{Transfer Characteristic}

The family of curves in figure 8 are typical of the pressure-frequency transfer characteristics of a capsule-oscillator combination. A number of diaphragms, made from the same roll of material and formed on the same die, showed essentially the same family. Curve Aa was obtained with the clamping: screws barely tight. Tightening the clamping screws with a torque screwdriver in 5 -in.-lb increments produced progressively higher sensitivities and improved linearity up to $20 \mathrm{in}$--lb of torque. Note that for 25 in.-lb the curve is quite unsymmetrical and the linear range is markedly reduced. A quite similar family of curves could be obtained by loosening all screws and progressively retightening them, but not by progressive loosening only.

Attempts have been made to determine the volume compliance of these diaphragms at the rest-point. The results indicate a range of about 0.002 to 0.008 $\mathrm{cm}^{3}$ per $\mathrm{d} / \mathrm{cm}^{2}$. 


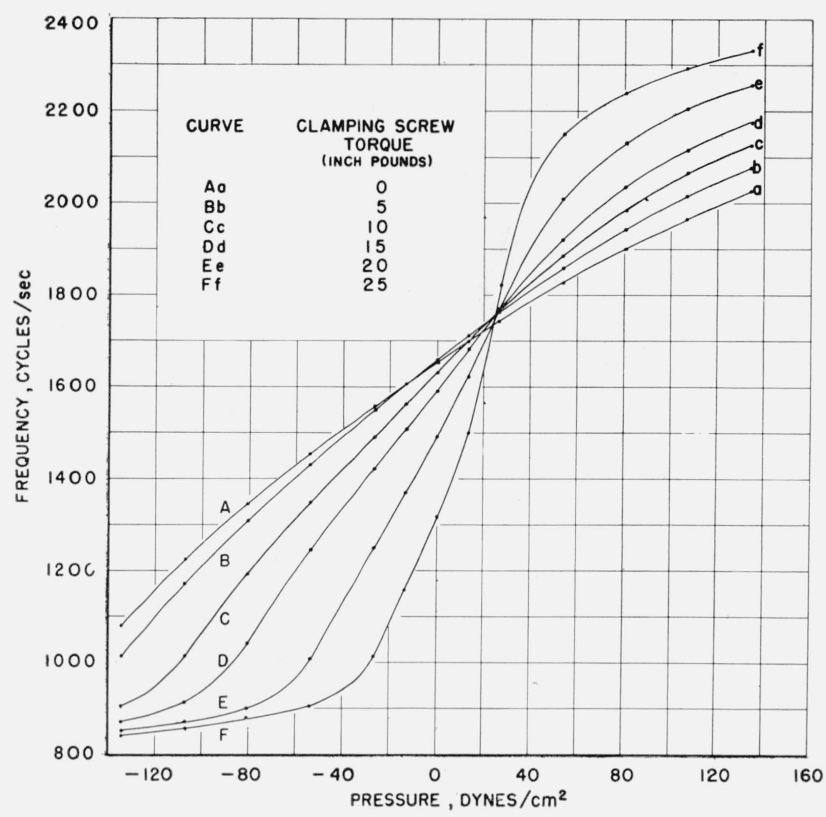

Figure 8. Curves showing the effect of clamping pressure, on a diaphragm, on the transfer characteristics of a capsuleoscillator combination.

\subsection{Serviceability}

The unmounted diaphragms had to be handled with considerable care. Injudicious flexing of an unmounted diaphragm impaired its performance, probably because the cupping was disturbed. Even edge damage limited to the portion of the diaphragm that was firmly clamped, altered the pressuredeflection characteristics. In practice, the diaphragms were never handled with the fingers or with tweezers but were manipulated on sheets of paper by tilting the paper to slide the diaphragm as required. Diaphragms could be picked up from a flat surface by slipping a sheet of paper under them and lifting the paper. When necessary, they could be grasped safely between thumb and finger at the center. Storage and shipment in paper envelopes with a cardboard stiffener against the back of the diaphragm has been quite successful, provided the surfaces of the envelope were protected from pressure. Prolonged storage of diaphragms at high humidity produced surface staining, which, though unsightly, had no detectable effect on performance.

Diaphragms have, on many occasions, been removed from a capsule and returned to it without detectable effect on the pressure-frequency curve.

Over 1,200 capsule-months of operation have shown that the diaphragms will remain stable in sensitivity and rest-point when subjected to alternating pressures up to $100 \mathrm{~d} / \mathrm{cm}^{2}$ at intervals of several minutes. The service life of an assembled capsule is known to exceed 2 years and appears to be indefinite. Observations over a period of months indicate that the sensitivity of a capsule may increase on the order of 5 percent a year.

No data is available on temporary or permanent changes in rest-point resulting from long sustained pressure difference across representative diaphragms. One series of experiments on a single diaphragm indicated that a momentary pressure of $4,000 \mathrm{~d} / \mathrm{cm}^{2}$ produced a detectable effect on the rest-point. The momentary application of $6,000 \mathrm{~d} / \mathrm{cm}^{2}$ shifted, at least temporarily, the pressure-frequency curve of the capsule-oscillator combination, but the diaphragm was judged to be still serviceable.

Washington, November 9, 1956. 